IoAnnis K. Argyros (Lawton, OK)

Santhosh George (Karnataka)

DAYa RAM SAHU (Varanasi)

\title{
EXTENSIONS OF KANTOROVICH-TYPE THEOREMS FOR NEWTON'S METHOD
}

Abstract. We extend the applicability of Newton's method, so we can approximate a locally unique solution of a nonlinear equation in a Banach space setting in cases not covered before. To achieve this, we find a more precise set containing the Newton iterates than in earlier works.

1. Introduction. The most used iteration for generating a sequence approximating a locally unique solution $x^{\star}$ of a nonlinear equation

$$
F(x)=0,
$$

is undoubtedly Newton's method defined for all $n=0,1,2, \ldots$ by

$$
x_{n+1}=x_{n}-F^{\prime}\left(x_{n}\right)^{-1} F\left(x_{n}\right),
$$

where $x_{0}$ is an initial point and $F: \Omega \subseteq X \rightarrow Y$ is a continuously Fréchet differentiable operator between Banach spaces $X$ and $Y$ and $\Omega$ is a convex set.

There is an extensive literature on local as well as semilocal Kantorovichtype convergence results for Newton's method [1-14]. However, the convergence domain for Newton's method is small in general. In the present study, we show how to extend the convergence domain without adding hypotheses in the already existing works. To achieve this we provide a more precise location, where the Newton iterates lie, leading to smaller Lipschitz functions.

2010 Mathematics Subject Classification: 65G99, 47H17, 49M15.

Key words and phrases: Newton's method, semilocal convergence, majorant function, restricted convergence domain.

Received 3 October 2017; revised 30 December 2017.

Published online 7 June 2019. 
The rest of the study is organized as follows: In Sections 2 and 3 , we present the semilocal convergence analysis of Newton's method 1.2 . Section 4 contains numerical examples.

2. Convergence analysis for Newton's method. In this section, we present the semilocal convergence analysis of Newton's method for Fréchet differentiable operators.

Let $L(X, Y)$ stand for the space of all bounded linear operators from a Banach space $X$ to a Banach space $Y$. Define the balls $U(x, \rho)=\{y \in \Omega$ : $\|x-y\|<\rho\}$ and $\bar{U}(x, \rho)=\{y \in \Omega:\|x-y\| \leq \rho\}$.

Definition 2.1. Let $F: \Omega \subseteq X \rightarrow Y$ be a continuously Fréchet differentiable operator. Let $x_{0} \in \Omega$ be such that $F^{\prime}\left(x_{0}\right)^{-1} \in L(Y, X)$. We say that $F^{\prime}$ satisfies the $L_{0}$-center Lipschitzian condition on $\Omega_{0}$ if

$$
\left\|F^{\prime}\left(x_{0}\right)^{-1}\left(F^{\prime}(x)-F^{\prime}\left(x_{0}\right)\right)\right\| \leq L_{0}(r)\left\|x-x_{0}\right\|
$$

for all $r \in[0, R]$ and all $x \in \Omega_{0}:=U\left(x_{0}, r\right) \cap \Omega$, where $R>0$ and $L_{0}:[0, R] \rightarrow \mathbb{R}_{+} \cup\{0\}$ is a continuous and non-decreasing function with $L_{0}(0)=0$.

Define

$$
\begin{aligned}
\bar{r}_{0} & =\sup \left\{t \in[0, R): L_{0}(t)<1\right\} \\
\Omega_{1} & =U\left(x_{0}, \bar{r}_{0}\right) \cap \Omega .
\end{aligned}
$$

Notice that

$$
\bar{r}_{0} \leq R \quad \text { and } \quad \Omega_{1} \subseteq \Omega_{0} .
$$

Definition 2.2. Let $F: \Omega \subseteq X \rightarrow Y$ be a continuously Fréchet differentiable operator. Let $x_{0} \in \Omega$ be such that $F^{\prime}\left(x_{0}\right)^{-1} \in L(Y, X)$. We say that $F^{\prime}$ satisfies the restricted L-Lipschitzian condition on $\Omega_{1}$ if

$$
\left\|F^{\prime}\left(x_{0}\right)^{-1}\left(F^{\prime}(x)-F^{\prime}(y)\right)\right\| \leq L(r)\|x-y\|
$$

for all $r \in\left[0, \bar{r}_{0}\right]$ and all $x, y \in \Omega_{1}$, where $L:\left[0, \bar{r}_{0}\right) \rightarrow \mathbb{R}_{+} \cup\{0\}$ is a continuous and non-decreasing function with $L(0)=0$.

It is convenient for the semilocal convergence analysis that follows to introduce functions $\varphi_{0}:[0, R] \rightarrow \mathbb{R}_{+} \cup\{0\}$ and $\varphi:\left[0, \bar{r}_{0}\right] \rightarrow \mathbb{R}_{+} \cup\{0\}$ defined by

$$
\begin{gathered}
\varphi_{0}(r)=b-r+\int_{0}^{r} L_{0}(t)(r-t) d t \\
\varphi(r)=b-r+\int_{0}^{r} L(t)(r-t) d t
\end{gathered}
$$

for some $b \geq 0$. 
We now give the semilocal convergence analysis of Newton's method using the preceding notation.

TheOREM 2.3. Let $F: \Omega \subseteq X \rightarrow Y$ be a continuously Fréchet differentiable operator satisfying the $L_{0}$-center Lipschitzian condition (2.1) on $\Omega_{0}$ and the restricted L-Lipschitz condition (2.5) on $\Omega_{1}$ for some $x_{0} \in \Omega$ such that $F^{\prime}\left(x_{0}\right)^{-1} \in L(Y, X)$ and $\left\|F^{\prime}\left(x_{0}\right)^{-1} F\left(x_{0}\right)\right\| \leq b$ for some $b \geq 0$. Moreover, suppose:

(i) The function $\varphi$ defined by (2.7) has a unique zero $r_{-}$in $\left[0, \bar{r}_{0}\right)$ such that $\varphi\left(\bar{r}_{0}\right) \leq 0$.

(ii) $L_{0}(r) \leq L(r)$ for all $\left[0, \bar{r}_{0}\right)$.

(iii) $\bar{U}\left(x_{0}, r_{-}\right) \subseteq \Omega$.

Then the following statements hold:

(a) The Newton sequence $\left\{s_{n}\right\}$ generated by

$$
s_{0}=0, \quad s_{n+1}=s_{n}-\frac{\varphi\left(s_{n}\right)}{\varphi^{\prime}\left(s_{n}\right)}, \quad n=0,1, \ldots,
$$

is well defined in $\left[0, r_{-}\right]$and converges monotonically to $r_{-}$.

(b) The Newton sequence $\left\{x_{n}\right\}$ generated by 1.2 is also well defined, remains in $\bar{U}\left(x_{0}, r_{-}\right)$and converges to a unique zero of $F$ in $U\left(x_{0}, \bar{r}_{0}\right)$. Moreover,

$$
\begin{aligned}
& \left\|x_{n}-x^{*}\right\| \leq r_{-}-s_{n}, \quad\left\|x_{n+1}-x_{n}\right\| \leq s_{n+1}-s_{n}, \\
& \left\|x_{n}-x^{*}\right\| \leq \mu^{2^{n}}\left(\bar{r}_{0}-s_{n}\right),
\end{aligned}
$$

where $\mu=\left\|x_{n}-x^{*}\right\| / \bar{r}_{0}$.

Proof. Simply repeat the corresponding proofs in [14], but use the estimate (see (2.1))

$$
\left\|F^{\prime}(x)^{-1} F\left(x_{0}\right)\right\| \leq-\frac{1}{\varphi^{\prime}\left(\left\|x-x_{0}\right\|\right)}
$$

instead of the less precise estimate

$$
\left\|F^{\prime}(x)^{-1} F\left(x_{0}\right)\right\| \leq-\frac{1}{\psi^{\prime}\left(\left\|x-x_{0}\right\|\right)}
$$

(see [14, (2.8) and (ii)]). Moreover notice that the iterates $\left\{x_{n}\right\}$ lie in $\Omega_{1}$, which is a more precise location than $\Omega_{0}$ (see (2.8)) used for the proof in [14, Proposition 4, p. 677].

We have the following useful alternative for the uniqueness part.

Proposition 2.4. Under the hypothesis of Theorem 2.3, further suppose that there exists $\gamma \in\left[r_{-1}, r_{0}\right)$ such that

$$
\frac{1}{2} L\left(r_{0}\right)\left(\gamma+r_{-1}\right)<1 .
$$

Then $x^{*}$ is the only zero of $F$ in $\Omega_{2}=\Omega \cap \bar{U}\left(x_{0}, \gamma\right)$. 
Proof. The proof uses the standard arguments [3] and condition (2.1) instead of condition (2.8) of 14 .

REMARK 2.5. (a) In order for us to compare the preceding results with the corresponding ones in 814 , let us consider the $L_{1}$-Lipschitzian condition on $\Omega_{0}$ (not on $\Omega_{1}$ ) with $L_{1}(0)=0$ and the corresponding majorant function $\psi$ (used in [14]). That is, we have

$$
\left\|F^{\prime}\left(x_{0}\right)^{-1}\left(F^{\prime}(x)-F^{\prime}(y)\right)\right\| \leq L_{1}(r)\|x-y\|
$$

for all $r \in[0, R]$ and all $x, y \in \Omega_{0}$, where

$$
\psi(r)=b-r+\int_{0}^{r} L_{1}(t)(r-t) d t .
$$

In view of (2.1), 2.5)-2.9), for each $r \in\left[0, r_{0}\right)$ we have

$$
\begin{aligned}
L_{0}(r) & \leq L_{1}(r), \\
L(r) & \leq L_{1}(r), \\
\varphi_{0}(r) & \leq \psi(r), \\
\varphi(r) & \leq \psi(r) .
\end{aligned}
$$

Define also the Newton iteration corresponding to $\psi$ by

$$
s_{0}=0, \quad s_{n+1}=s_{n}-\frac{\psi\left(s_{n}\right)}{\psi^{\prime}\left(s_{n}\right)}, \quad n=0,1, \ldots
$$

Let $s_{-}$be the unique zero of the function $\psi$ in $[0, R)$ such that $\psi(R) \leq 0$. These conditions imply the corresponding conditions of Theorem 2.3, but not necessarily vice versa. Hence, the new sufficient semilocal convergence conditions are at least just as weak.

Concerning the comparison between the majorizing sequences $\left\{r_{n}\right\}$ and $\left\{s_{n}\right\}$, further suppose that for all $u, v \in[0, R]$ with $u \leq v$,

$$
-\frac{\varphi(u)}{\varphi^{\prime}(u)} \leq-\frac{\psi(v)}{\psi^{\prime}(v)} \text {. }
$$

Then a simple inductive argument using 2.10 -2.13 shows, for $n=0,1, \ldots$,

$$
\begin{aligned}
r_{n} & \leq s_{n}, \\
r_{n+1}-r_{n} & \leq s_{n+1}-s_{n}, \\
r_{-} & \leq s_{-} .
\end{aligned}
$$

Moreover, strict inequality may hold in 2.15) (for $n=2,3, \ldots$ ) and in 2.16 ) (for $n=1,2, \ldots$ ) if it does in 2.11) or (2.10).

(b) It follows from the proof of Theorem 2.3 that the sequence $\left\{q_{n}\right\}$ defined by

$$
q_{0}=0, \quad q_{1}=q_{0}-\frac{\varphi\left(q_{0}\right)}{\varphi^{\prime}\left(q_{0}\right)}, \quad q_{n+1}=q_{n}-\frac{\varphi\left(q_{n}\right)}{\varphi^{\prime}\left(q_{n}\right)}, \quad n=1,2, \ldots,
$$


is a more precise majorizing sequence than $\left\{r_{n}\right\}$ which converges under the same hypotheses such that

$$
\begin{aligned}
q_{n} & \leq r_{n}, \\
q_{n+1}-q_{n} & \leq r_{n+1}-r_{n}, \\
q_{-} & =\lim _{n \rightarrow \infty} q_{n}=r_{-} .
\end{aligned}
$$

(c) If condition (ii) of Theorem 2.3 is not satisfied, i.e.

$$
L(r)<L_{0}(r),
$$

then $\varphi_{0}$ can replace $\varphi$ in Theorem 2.3.

(d) The uniqueness given in Proposition 2.4 also improves the corresponding one in [14], where $L_{1}$ was used instead of the more precise $L_{0}$ (see (2.10).

It is worth noticing that in practice the computation of the original function $L_{1}$ requires the computation of the functions $L_{0}$ and $L$ as special cases. That is, no hypotheses additional to [14] are needed to obtain these improvements.

3. Semilocal convergence II. In this section, we study the convergence of Newton's method for operators $F$ that are $p \geq 2$ ( $p$ an integer) times Fréchet differentiable.

Proposition 3.1. Let $F: \Omega \subseteq X \rightarrow Y$ be a $p \geq 2$ times continuously Fréchet differentiable operator satisfying the $L_{0}$-center Lipschitzian condition on $\Omega_{0}$ for some $x_{0} \in \Omega$ such that $F^{\prime}\left(x_{0}\right)^{-1} \in L(Y, X)$. Moreover, suppose:

(i) $\left\|F^{\prime}\left(x_{0}\right)^{-1} F\left(x_{0}\right)\right\| \leq b$ and $\left\|F^{\prime}\left(x_{0}\right)^{-1} F^{(i)}\left(x_{0}\right)\right\| \leq c_{i}, i=2, \ldots, p$, for some $b, c_{i} \geq 0$.

(ii) There exists a continuous non-decreasing function $L^{(p)}:\left[0, \bar{r}_{0}\right) \rightarrow \mathbb{R}_{+}$ $\cup\{0\}$ such that

$$
\left\|F^{\prime}\left(x_{0}\right)^{-1}\left(F^{(p)}(y)-F^{(p)}(x)\right)\right\| \leq L^{(p)}(r)\|y-x\|
$$

for all $r \in\left[0, r_{0}\right]$ and all $x, y \in \Omega_{1}$.

(iii) The function $\varphi:\left[0, \bar{r}_{0}\right] \rightarrow \mathbb{R}_{+} \cup\{0\}$ defined by

$$
\varphi(r)=b-r+c_{2} \frac{r^{2}}{2 !}+\cdots+c_{p} \frac{r^{p}}{p !}+\int_{0}^{r} L^{(p)}(t) \frac{(r-t)^{p}}{p !} d t
$$

has a unique zero $r_{-}$in $\left[0, \bar{r}_{0}\right)$ and $\varphi\left(\bar{r}_{0}\right) \leq 0$, where

$$
L(r)=\varphi^{\prime \prime}(r)=c_{2}+\cdots+c_{p} \frac{r^{p-2}}{(p-2) !}+\int_{0}^{r} L^{(p)}(t) \frac{(r-t)^{p-2}}{(p-2) !} d t .
$$


(iv) $L_{0}(r) \leq L(r)$ for all $r \in\left[0, \bar{r}_{0}\right)$,

(v) $\bar{U}\left(x_{0}, r_{-}\right) \subseteq \Omega$.

Then the conclusions of Theorem 2.3 hold.

Proof. Let $r_{x}=\left\|x-x_{0}\right\|$. It is a straightforward application of Taylor's theorem to show that in both cases $p=2$ and $p \geq 3$ we have

$$
\left\|F^{\prime}\left(x_{0}\right)^{-1} F^{\prime \prime}(x)\right\| \leq L\left(r_{x}\right) .
$$

Hence, $L$ satisfies the hypotheses of Theorem 2.3.

Proposition 3.2. Let $F: \Omega \subseteq X \rightarrow Y$ be an infinitely many times continuously Fréchet differentiable operator satisfying the $L_{0}$-center Lipschitzian condition on $\Omega_{0}$ for some $x_{0} \in \Omega$ such that $F^{\prime}\left(x_{0}\right)^{-1} \in L(Y, X)$. Moreover, suppose:

(i) $\left\|F^{\prime}\left(x_{0}\right)^{-1} F\left(x_{0}\right)\right\| \leq b$ and $\left\|F^{\prime}\left(x_{0}\right)^{-1} F^{i}\left(x_{0}\right)\right\| \leq c_{i}$, $i \geq 2$, for some $b, c_{i} \geq 0$.

(ii) The function $\varphi:\left[0, \bar{r}_{0}\right] \rightarrow \mathbb{R}_{+} \cup\{0\}$ is defined by

$$
\varphi(r)=b-r+\sum_{p \geq 2} c_{p} \frac{r^{p}}{p !}
$$

assuming that the series converges and has a unique zero $r_{-}$in $\left[0, \bar{r}_{0}\right)$.

(iii) $L_{0}(r) \leq L(r)$ for all $r \in\left[0, \bar{r}_{0}\right)$, where

$$
L(r)=\varphi^{\prime \prime}(r)=\sum_{p \geq 2} c_{p} \frac{r^{p-2}}{(p-2) !} .
$$

(iv) $\bar{U}\left(x_{0}, r_{-}\right) \subset \Omega$.

Then the conclusions of Theorem 2.3 hold.

Proof. By the expansion of $F^{\prime \prime}$ at $x_{0}$, we again get

$$
\left\|F^{\prime}\left(x_{0}\right)^{-1} F^{\prime \prime}(x)\right\| \leq L\left(\left\|x-x_{0}\right\|\right)
$$

for all $x \in \Omega_{1}$. Hence $L$ satisfies the hypotheses of Theorem 2.3.

4. Numerical examples. We present two numerical examples, where the function $\psi$ in 2.9 has no real zero. Hence the older results do not apply 5 14, but the function $\varphi$ has solutions, so the new results apply to solve equations.

In both examples, $L_{0}, L$ and $L_{1}$ are constant functions. Notice that in this case the functions $\varphi_{0}, \varphi$ and $\psi$ are reduced to

$$
\varphi_{0}(r)=\frac{L_{0}}{2} r^{2}-r+b, \quad \varphi(r)=\frac{L}{2} r^{2}-r+b, \quad \psi(r)=\frac{L_{1}}{2} r^{2}-r+n .
$$

Therefore, the equations

$$
\varphi_{0}(r)=0, \quad \varphi(r)=0, \quad \psi(r)=0
$$


each have real solutions provided that the respective Newton-Kantorovichtype conditions 8

$$
\begin{array}{r}
2 L_{0} b \leq 1, \\
2 L b \leq 1, \\
2 L_{1} b \leq 1
\end{array}
$$

hold.

Example 4.1. Let $X=Y=\mathbb{R}, x_{0}=1, \Omega=\left\{x:\left|x-x_{0}\right| \leq 1-\beta\right\}$, $\beta \in[0,1 / 2), R=1-\beta, \bar{r}_{0}=1 / L_{0}$. Define a function $F$ on $\Omega$ by

$$
F(x)=x^{3}-\beta .
$$

Using the hypotheses of Theorem 2.3 , we get

$$
b=\frac{1-\beta}{3},
$$

and

$$
\begin{aligned}
\left|F^{\prime}\left(x_{0}\right)^{-1}\left(F^{\prime}(x)-F^{\prime}\left(x_{0}\right)\right)\right| & =\left|x^{2}-x_{0}^{2}\right|=\left|x+x_{0}\right|\left|x-x_{0}\right| \\
& \left.=\left|\left(x-x_{0}\right)+2\right| x_{0} \mid\right)\left|x-x_{0}\right| \\
& \leq\left(\left|x-x_{0}\right|+2\left|x_{0}\right|\right)\left|x-x_{0}\right| \\
& \leq(1-\beta+2)\left|x-x_{0}\right|=(3-\beta)\left|x-x_{0}\right|
\end{aligned}
$$

for each $x \in \Omega_{0}$. So, we can choose $L_{0}=3-\beta$. Moreover, we have

$$
\begin{aligned}
\left|F^{\prime}\left(x_{0}\right)^{-1}\left(F^{\prime}(x)-F^{\prime}(y)\right)\right| & =\left|x^{2}-y^{2}\right|=|x+y||x-y| \\
& =\left|\left(x-x_{0}\right)+\left(y-x_{0}\right)+2 x_{0}\right||x-y| \\
& \leq\left(\left|x-x_{0}\right|+\left|y-x_{0}\right|+2\left|x_{0}\right|\right)|x-y| \\
& \leq(2(1-\beta)+2)|x-y|=2(1-\beta)|x-y|
\end{aligned}
$$

for all $x, y \in \Omega_{0}$, so we can choose $L_{1}=2(2-\beta)$. Furthermore, for each $x, y \in \Omega_{1}=U\left(x, \bar{r}_{0}\right) \cap U\left(x_{0}, 1-\beta\right)=U\left(x_{0}, \bar{r}_{0}\right)$ (since $\bar{r}_{0}<1-\beta$ ) we obtain

$$
\begin{aligned}
\left|F^{\prime}\left(x_{0}\right)^{-1}\left(F^{\prime}(x)-F^{\prime}(y)\right)\right| & \leq\left(\left|x-x_{0}\right|+\left|y-x_{0}\right|+2\left|x_{0}\right|\right)|x-y| \\
& \leq\left(2+2 \bar{r}_{0}\right)|x-y|=2\left(1+\frac{1}{3-\beta}\right)|x-y|,
\end{aligned}
$$

so we can choose $L(r)=2\left(1+\frac{1}{3-\beta}\right)$. Notice that

$$
L_{0}<L<L_{1} \quad \text { and } \quad \bar{r}_{0}<R \quad \text { for all } \beta \in[0,1 / 2) .
$$

The Newton-Kantorovich condition 4.3 is not satisfied, since

$$
\frac{4}{3}(1-\beta)(2-\beta)>1 \quad \text { for all } \beta \in[0,1 / 2) .
$$

Hence, there is no guarantee that Newton's method 1.2 converges to 
$x^{*}=\sqrt[3]{\beta}$, starting at $x_{0}=1$. However, our corresponding condition 4.2 is true for all $\beta \in I=[0.4619832,1 / 2)$. Hence, the conclusions of our Theorem 2.3 can be applied to solve the equation $F(x)=0$ for all $\beta \in I$.

EXAmPLE 4.2. Let $X=Y=\mathcal{C}[0,1]$, the space of continuous real-valued functions defined on $[0,1]$. We shall use the max-norm. Let $\Omega=\{x \in X$ : $\|x\| \leq R\}$ such that $R>0$. Define $F$ on $\Omega$ by 7,10

$$
F(x)(s)=x(s)-f(s)-\delta \int_{0}^{1} K(s, t) x(t)^{3} d t, \quad x \in X, s \in[0,1],
$$

where $f \in X$ is a given function, $\delta$ is a real constant and the kernel $K$ is the Green's function defined by

$$
K(s, t)= \begin{cases}(1-s) t & \text { if } t \leq s, \\ s(1-t) & \text { if } s \leq t .\end{cases}
$$

It follows from (4.6) that, for each $x \in \Omega, F^{\prime}(x)$ is a linear operator defined by

$$
\left[F^{\prime}(x)(v)\right](s)=v(s)-3 \delta \int_{0}^{1} K(s, t) x(t)^{2} v(t) d t, \quad v \in X, s \in[0,1] .
$$

Let us choose $x_{0}(s)=f(s)=1$. It follows that $\left\|I-F^{\prime}\left(x_{0}\right)\right\| \leq 3|\delta| / 8$. If $|\delta|<8 / 3$, then $F^{\prime}\left(x_{0}\right)^{-1}$ exists and

$$
\left\|F^{\prime}\left(x_{0}\right)^{-1}\right\| \leq \frac{8}{8-3|\delta|} .
$$

We also get $\left\|F\left(x_{0}\right)\right\| \leq|\delta| / 8$, so

$$
b=\left\|F^{\prime}\left(x_{0}\right)^{-1} F\left(x_{0}\right)\right\| \leq \frac{|\delta|}{8-3|\delta|} .
$$

Moreover, for $x, y \in \Omega$, we obtain

and

$$
\left\|F^{\prime}(x)-F^{\prime}(y)\right\| \leq \frac{1+3|\delta|\|x+y\|}{8}\|x-y\| \leq \frac{1+6 R|\delta|}{8}\|x-y\|
$$

$$
\left\|F^{\prime}(x)-F^{\prime}(1)\right\| \leq \frac{1+3|\delta|(\|x\|+1)}{8}\|x-1\| \leq \frac{1+3|\delta|(1+R)}{8}\|x-1\| .
$$

Choosing $\delta=1.175$ and $R=2$, we have $b=0.26257 \ldots, L_{1}=2.76875 \ldots$, $L_{0}=1.8875 \ldots, 1 / L_{0}=0.529801 \ldots$ and $L=1.47314 \ldots$.

Using these values, we find that condition 4.3 is not satisfied, since

$$
1.4539813>1 .
$$

However, our condition 4.2 is satisfied, since

$$
0.7736047<1 \text {. }
$$

Hence, the convergence of Newton's method is guaranteed by Theorem 2.3. 


\section{References}

[1] I. K. Argyros, Computational Theory of Iterative Methods, Stud. Comput. Math. 15, Elsevier, Amsterdam, 2007.

[2] I. K. Argyros, On the Newton-Kantorovich hypothesis for solving equations, J. Comput. Appl. Math. 169 (2004), 315-332.

[3] I. K. Argyros, A unifying local-semilocal convergence analysis and applications for two-point Newton-like methods in Banach space, J. Math. Anal. Appl. 298 (2004), 374-397.

[4] I. K. Argyros and S. Hilout, Weaker conditions for the convergence of Newton's method, J. Complexity 28 (2012), 364-387.

[5] J. E. Dennis, Jr., Toward a unified convergence theory for Newton-like methods, in: Nonlinear Functional Analysis and Applications (Madison, WI, 1970), Academic Press, New York, 1971, 425-472.

[6] P. Deuflhard and G. Heindl, Affine invariant convergence theorems for Newton's method and extensions to related methods, SIAM J. Numer. Anal. 16 (1979), 1-10.

[7] J. A. Ezquerro and M. A. Hernández, New iterations of $R$-order four with reduced computational cost, BIT 49 (2009), 325-342.

[8] L. V. Kantorovich and G. P. Akilov, Functional Analysis, Pergamon Press, Oxford, 1982.

[9] Á. A. Magreñán, A new tool to study real dynamics: the convergence plane, Appl. Math. Comput. 248 (2014), 215-224.

[10] Á. A. Magreñán and I. K. Argyros, Improved convergence analysis for Newton-like methods, Numer. Algorithms 71 (2016), 811-826.

[11] F. A. Potra and V. Pták, Nondiscrete Induction and Iterative Processes, Res. Notes in Math. 103, Pitman (Advanced Publishing Program), Boston, MA, 1984.

[12] P. D. Proinov, New general convergence theory for iterative processes and its applications to Newton-Kantorovich type theorems, J. Complexity 26 (2010), 3-42.

[13] W. C. Rheinboldt, A unified convergence theory for a class of iterative processes, SIAM J. Numer. Anal. 5 (1968), 42-63.

[14] P. P. Zabrejko and D. F. Nguen, The majorant method in the theory of NewtonKantorovich approximations and the Pták error estimates, Numer. Funct. Anal. Optim. 9 (1987), 671-684.

Ioannis K. Argyros

Department of Mathematical Sciences

Cameron University

Lawton, OK 73505, U.S.A.

ORCID: 0000-0003-1609-3195

E-mail: iargyros@cameron.edu

Daya Ram Sahu

Department of Mathematics

Banaras Hindu University

Varanasi 221005, India

E-mail: drsahudr@gmail.com
Santhosh George Department of Mathematical and Computational Sciences National Institute of Technology Karnataka, India ORCID: 0000-0002-3530-5539 E-mail: sgeorge@nitk.ac.in 
